\title{
From gradient damage laws to Griffith's theory of crack propagation
}

\author{
Paul Sicsic • Jean-Jacques Marigo
}

accepted 12 september 2012

\begin{abstract}
This paper is devoted to the comparison of the evolution of damage governed by a gradient damage model with the evolution of a crack predicted by Griffith's law. The analysis is made in a two-dimensional setting, assuming that damage is concentrated inside thin bands whose width is proportional to the internal length of the material. Taking advantage of the variational formulation based on the three principles of irreversibility, stability and energy balance, one introduces a generalized Rice path integral which contains terms involving the gradient of damage. Assuming that the internal length of the material is small by comparison with the dimension of the body, a separation of scales is achieved. Owing to the energy balance and the stability condition, one first proves some properties of this path integral with respect to the path. Then, one shows that the evolution of the damage zone is governed by Griffith's law, the dissipated surface energy being given by the energy dissipated in the damage process zone.
\end{abstract}

Keywords Damage, Gradient Model, Fracture, Variational Methods, Path integral

Mathematics Subject Classification (2000) 74R10, 49J40, 26A45, 47J30

\section{Introduction}

In the variational approach to fracture (Ambrosio and Tortorelli 1990; Bourdin et al. 2008) the regularized functional introduced to approximate the total energy associated with Griffith's assumption on the surface energy can be interpreted as a non local damage model in the spirit of those developed by Comi (1999), Lorentz and

Paul Sicsic

Lafarge Centre de Recherche, 95 Rue de Montmurier 38290 St-Quentin-Fallavier, France

Paul Sicsic · Jean-Jacques Marigo

Laboratoire de Mécanique des Solides, Ecole Polytechnique, F91128 Palaiseau Cedex

E-mail: sicsic@lms.polytechnique.fr

E-mail: marigo@lms.polytechnique.fr 
Andrieux (1999) or Pham and Marigo (2010b). These damage gradient models contain an internal length $\eta$ which can be considered as a material characteristic. Whenever this length is small by comparison with the dimensions of the body, it can be proven by Gamma-convergence arguments (Ambrosio and Tortorelli 1990; Braides 2002) that the global minimum of the regularized functional converges (in an appropriate sense) to the global minimum of Griffith's functional. This result can be considered as a fundamental link between damage and fracture mechanics. However it deserves to be improved and generalized by removing the concept of global minimization of the energy which cannot be considered as a good physical principle. Indeed, this type of global minimization allows jumps from one state to the other without considering the presence of energy barriers. Therefore, we will replace the global minimization principle by a stability condition which can be considered as a local minimization principle. One is then interested whether the evolution of damage governed by such a weaker condition remains close to the one prescribed by Griffith's law when the internal length is small. The same question appears when considering numerical tests based on an alternate minimization algorithm Bourdin (2007a,b). Indeed, such an algorithm does not converge necessarily to a global minimizer of the energy, but only to a stationary state. The issue is to compare the evolution given by the numerical computations with Griffith's law. Practically all the numerical simulations show that, after a stage of nucleation, the damage concentrates in bands whose width is of the order of the characteristic length $\eta$ of the material Bourdin (2007a,b). Moreover, except at the tip of the damage zone, the damage profile in the direction orthogonal to the band is practically the one given by a one-dimensional analysis Pham and Marigo (2012a). These two properties (a thin damage band with an optimal profile) will be the basic assumptions of our analysis from which we can establish the link with Griffith's law. The last major issue in order to achieve this task is to give a sense to the concepts of energy release rate and of critical energy release rate, that is to say, to introduce correctly the basic quantities $G$ and $G_{c}$ entering in Griffith's law in the setting of our damage law. Specifically, in Griffith's theory, $G$ is defined by assuming that the material has a purely elastic behavior. Then by virtue of Irwin's formula one can relate the energy release rate to the singularity at the tip of the crack and therefore to the stress intensity factors. In the context of the present damage law, it turns out that there is no more singularities of the stresses (but the gradient of damage can be singular!), the stresses remain bounded. Besides, in Griffith's theory, $G_{C}$ is a given material constant characterizing the energy associated with surfaces of discontinuity whereas in our damage approach the parameters characterizing the inelastic behavior of the material are the critical stress $\sigma_{c}$ and the internal length $\eta$. Accordingly, we will define $G$ with the help of a two-scale approach and we will define $G_{c}$ as the energy dissipated by creating the optimal damage profile.

Specifically the paper is organized as follows. In Section 2.1 we recall the gradient damage model and the three physical principles governing the evolution law. After introducing fundamental assumptions, we establish some general properties of the damage evolution in Sections 2.2 and 2.3. Then, in Section 3, we proceed to the separation of scales by assuming that the internal length $\eta$ is small by comparison with the length of the damage band and any other structural dimension. We first study the damage problem far from the crack band and the crack tip. That allows us to define $G$, see Section 3.1. In Section 3.2, after studying the damage problem in the damage band (far enough from its tip), we identify $G_{c}$ with the dissipated energy in any unit of length of the damage band. In Section 3.3, we analyze the damage problem in the neighborhood of the crack tip. That leads to the introduction of a generalized Rice path-integral which is studied in Section 3.4. We establish in particular some properties relative to its path dependence. We are then in a position to conclude and to make the link between the propagation law of the damage band and Griffith's law (Section 3.5). 


\section{The gradient damage model and fundamental assumptions}

\subsection{Setting of the gradient damage model}

We simply recall here the main steps of the construction of a gradient damage model by a variational approach, the reader interested by more details should refer to Pham and Marigo (2010a) and Pham and Marigo (2010b). For the sake of simplicity, we will restrict the analysis to a plane strain setting, but the major part of the analysis remains valid in 3D. Let us consider a homogeneous body whose reference configuration is the open connected bounded set $\Omega \subset \mathbb{R}^{2}$ of characteristic size $L$. This body is made of a strongly brittle damaging material whose behavior is defined as follows:

1. The damage parameter is a scalar which can only grow from 0 to $1, \alpha=0$ denoting the undamaged state and $\alpha=1$ the completely damaged state.

2. The state of the volume element is characterized by the triplet $(\varepsilon, \alpha, \mathbf{g})$ where $\varepsilon, \alpha$ and $\mathbf{g}$ denote respectively the strain tensor, the damage parameter and the gradient of damage vector $(\mathbf{g}=\nabla \alpha)$.

3. The bulk energy density of the material is the state function $W:(\varepsilon, \alpha, \mathbf{g}) \mapsto W(\varepsilon, \alpha, \mathbf{g})$. Therefore, the material behavior is non local in the sense that it depends on the gradient of damage. To simplify the presentation, we will only consider behaviors such that the bulk energy density is the sum of three terms: the stored elastic energy $\psi(\varepsilon, \alpha)$, the local part of the dissipated energy by damage $\mathbf{w}(\alpha)$ and its non local part $\frac{1}{2} \mathbf{w}_{1} \eta^{2} \mathbf{g} \cdot \mathbf{g}$,

$$
W(\varepsilon, \alpha, \mathbf{g})=\psi(\varepsilon, \alpha)+\mathrm{w}(\alpha)+\frac{1}{2} \mathrm{w}_{1} \eta^{2} \mathbf{g} \cdot \mathbf{g},
$$

each of these terms enjoying the following properties:

(a) The elastic energy reads as

$$
\psi(\varepsilon, \alpha)=\frac{1}{2} \mathrm{~A}(\alpha)\left(\varepsilon-\varepsilon^{0}\right) \cdot\left(\varepsilon-\varepsilon^{0}\right),
$$

where $\varepsilon^{0}$ is a given pre-strain and $\mathrm{A}(\alpha)$ is the stiffness tensor of the material in its damaged state $\alpha$. The stiffness function $\alpha \mapsto \mathrm{A}(\alpha)$ decreases from $\mathrm{A}_{0}$ to 0 when $\alpha$ grows from 0 to 1 .

(b) The local dissipated energy density is a positive increasing function of $\alpha$, increasing from 0 when $\alpha=0$ to a finite positive value $\mathrm{w}_{1}$ when $\alpha=1$. Therefore $\mathrm{w}_{1}$ represents the energy dissipated during a complete, homogeneous damage process of a volume element: $w_{1}=w(1)$.

(c) The non local dissipated energy density is assumed to be a quadratic function of the gradient of damage. Since the damage parameter is dimensionless and by virtue of the above definition of $w_{1}, \eta$ has the dimension of a length. Accordingly, $\eta$ can be considered as an internal length characteristic of the material while having always in mind that the definition of $\eta$ depends on the normalizations associated with the choices of the critical value 1 for $\alpha$ and $w(1)$ for the multiplicative factor.

4. The dual quantities associated with the state variables are respectively the stress tensor $\boldsymbol{\sigma}$, the energy release rate density $\mathrm{Y}$ and the damage flux vector $\mathbf{q}$ :

$$
\boldsymbol{\sigma}=\frac{\partial W}{\partial \boldsymbol{\varepsilon}}(\varepsilon, \alpha, \mathbf{g}), \quad \mathbf{Y}=-\frac{\partial W}{\partial \alpha}(\varepsilon, \alpha, \mathbf{g}), \quad \mathbf{q}=\frac{\partial W}{\partial \mathbf{g}}(\varepsilon, \alpha, \mathbf{g}) .
$$

Accordingly, these dual quantities are given by the following functions of state:

$$
\boldsymbol{\sigma}=\mathrm{A}(\alpha)\left(\varepsilon-\varepsilon^{0}\right), \quad \mathrm{Y}=-\frac{1}{2} \mathrm{~A}^{\prime}(\alpha)\left(\varepsilon-\varepsilon^{0}\right) \cdot\left(\varepsilon-\varepsilon^{0}\right)-\mathrm{w}^{\prime}(\alpha), \quad \mathbf{q}=\mathrm{w}_{1} \eta^{2} \mathbf{g},
$$

where the prime denotes the derivative with respect to $\alpha$. 
The underlying local behavior is characterized by the function $W_{0}$ defined by $W_{0}(\varepsilon, \alpha)=W(\varepsilon, \alpha, \mathbf{0})$. We assume that it corresponds to a strongly brittle material, see (Pham and Marigo 2012a, Hypothesis 1). That means specifically that the material has a softening behavior but also that the energy dissipated during a process where the damage parameter grows from 0 to 1 is finite. The latter property is ensured by the fact that $\mathrm{w}(1)<+\infty$. The former one requires that the elastic domain in the strain space $\mathcal{R}(\alpha)$ is an increasing function of $\alpha$ while the elastic domain in the stress space $\mathcal{R}^{*}(\alpha)$ is a decreasing function of $\alpha$. Those elastic domains are defined by

$$
\mathcal{R}(\alpha)=\left\{\varepsilon \in \mathbb{M}_{s}: \frac{\partial W_{0}}{\partial \alpha}(\varepsilon, \alpha) \geq 0\right\}, \quad \mathcal{R}^{*}(\alpha)=\left\{\boldsymbol{\sigma} \in \mathbb{M}_{s}: \frac{\partial W_{0}^{*}}{\partial \alpha}(\boldsymbol{\sigma}, \alpha) \leq 0\right\}
$$

where $W_{0}^{*}(\boldsymbol{\sigma}, \alpha)=\sup _{\boldsymbol{\varepsilon} \in \mathbb{M}_{s}}\left\{\boldsymbol{\sigma} \cdot \boldsymbol{\varepsilon}-W_{0}(\varepsilon, \alpha)\right\}$ and $\mathbb{M}_{s}$ denotes the space of symmetric tensors.

In the present context, one gets

$$
W_{0}(\varepsilon, \alpha)=\frac{1}{2} \mathrm{~A}(\alpha)\left(\varepsilon-\varepsilon^{0}\right) \cdot\left(\varepsilon-\varepsilon^{0}\right)+\mathrm{w}(\alpha)
$$

and hence

$$
W_{0}^{*}(\boldsymbol{\sigma}, \alpha)=\boldsymbol{\sigma} \cdot \boldsymbol{\varepsilon}^{0}+\frac{1}{2} \mathrm{~S}(\alpha) \boldsymbol{\sigma} \cdot \boldsymbol{\sigma}-\mathrm{w}(\alpha),
$$

where the compliance tensor $\mathrm{S}(\alpha)=\mathrm{A}(\alpha)^{-1}$ increases from $\mathrm{S}_{0}$ to infinity when $\alpha$ grows from 0 to 1 . Accordingly, the elastic domains $\mathcal{R}(\alpha)$ and $\mathcal{R}^{*}(\alpha)$ now read

$$
\mathcal{R}(\alpha)=\left\{\varepsilon \in \mathbb{M}_{s}:-\frac{1}{2} \mathrm{~A}^{\prime}(\alpha)\left(\varepsilon-\varepsilon^{0}\right) \cdot\left(\varepsilon-\varepsilon^{0}\right) \leq \mathrm{w}^{\prime}(\alpha)\right\}, \quad \mathcal{R}^{*}(\alpha)=\left\{\boldsymbol{\sigma} \in \mathbb{M}_{s}: \frac{1}{2} \mathrm{~S}^{\prime}(\alpha) \boldsymbol{\sigma} \cdot \boldsymbol{\sigma} \leq \mathrm{w}^{\prime}(\alpha)\right\} .
$$

In order for the softening properties to be satisfied, the functions $\alpha \mapsto \mathrm{A}^{\prime}(\alpha) / \mathrm{w}^{\prime}(\alpha)$ and $\alpha \mapsto \mathrm{S}^{\prime}(\alpha) / \mathrm{w}^{\prime}(\alpha)$ must be increasing. The critical stress $\sigma_{c}$ in a uniaxial tensile test such that $\boldsymbol{\sigma}=\sigma_{c} \mathbf{e}_{1} \otimes \mathbf{e}_{1}$ is then given by

$$
\sigma_{c}=\sqrt{\frac{2 \mathrm{w}^{\prime}(0)}{\mathrm{S}_{1111}^{\prime}(0)}}
$$

The body is submitted to a time dependent loading which consists of a density of volume forces $\mathbf{f}_{t}$, a density of surface forces $\mathbf{F}_{t}$ prescribed on the part $\partial_{N} \Omega$ of the boundary and prescribed displacements $\mathbf{U}_{t}$ on the complementary part $\partial_{D} \Omega$ of the boundary, $t$ denoting the time parameter. The potential of the given external forces at time $t$ can read as the following linear form $\mathcal{W}_{t}^{e}$ defined on the set $\mathcal{C}_{t}$ of kinematically admissible displacement fields

with

$$
\mathcal{W}_{t}^{e}(\mathbf{v}):=\int_{\Omega} \mathbf{f}_{t} \cdot \mathbf{v} \mathrm{d} x+\int_{\partial_{N} \Omega} \mathbf{F}_{t} \cdot \mathbf{v} \mathrm{d} s
$$

$$
\mathcal{C}_{t}:=\left\{\mathbf{v}: \mathbf{v}=\mathbf{U}_{t} \text { on } \partial_{D} \Omega\right\}
$$

The law of evolution of the damage in the body is written in a variational form and based on the definition of the total energy of the body associated with admissible states. Specifically, if $(\mathbf{v}, \beta)$ denotes a pair of admissible displacement and damage fields at time $t$, i.e. if $\mathbf{v} \in \mathcal{C}_{t}$ and $\beta \in \mathcal{D}$ with

$$
\mathcal{D}:=\{\beta: 0 \leq \beta \leq 1 \text { in } \Omega\},
$$

then the total energy of the body at time $t$ in this state is given by

$$
\mathcal{P}_{t}(\mathbf{v}, \beta):=\int_{\Omega} W(\varepsilon(\mathbf{v}), \beta, \nabla \beta) \mathrm{d} x-\mathcal{W}_{t}^{e}(\mathbf{v})
$$

where $\boldsymbol{\varepsilon}(\mathbf{v})$ denotes the symmetrized gradient of $\mathbf{v}$. 
Hypothesis 1 (The damage evolution law) Following the variational approach presented in Pham and Marigo (2010a) and Pham and Marigo (2010b), the evolution of the damage in the body is governed by the three principles of irreversibility, stability and energy balance. Specifically these conditions read as follows:

1. Irreversibility: $t \mapsto \alpha_{t}$ must be non decreasing and, at each time $t \geq 0, \alpha_{t} \in \mathcal{D}$.

2. Stability: At each time $t>0$, the real state $\left(\mathbf{u}_{t}, \alpha_{t}\right)$ must be stable in the sense that for all $\mathbf{v} \in \mathcal{C}_{t}$ and all $\beta \in \mathcal{D}$ such that $\beta \geq \alpha_{t}$, there exists $\bar{h}>0$ such that for all $h \in[0, \bar{h}]$

$$
\mathcal{P}_{t}\left(\mathbf{u}_{t}+h\left(\mathbf{v}-\mathbf{u}_{t}\right), \alpha_{t}+h\left(\beta-\alpha_{t}\right) \geq \mathcal{P}_{t}\left(\mathbf{u}_{t}, \alpha_{t}\right) .\right.
$$

3. Energy balance: At each time $t>0$ the following energy balance must hold:

$$
\mathcal{P}_{t}\left(\mathbf{u}_{t}, \alpha_{t}\right)=\mathcal{P}_{0}\left(\mathbf{u}_{0}, \alpha_{0}\right)+\int_{0}^{t}\left(\int_{\Omega} \boldsymbol{\sigma}_{s} \cdot \varepsilon\left(\dot{\mathbf{U}}_{s}\right) \mathrm{d} x-\mathcal{W}_{s}^{e}\left(\dot{\mathbf{U}}_{s}\right)-\dot{\mathcal{W}}_{s}^{e}\left(\mathbf{u}_{s}\right)\right) \mathrm{d} s .
$$

In (11), $\alpha_{0}$ denotes the given damage state at the beginning of the loading process whereas $\mathbf{u}_{0}$ is the associated displacement field obtained by solving the elastostatic problem at time $0: \mathbf{u}_{0}=\operatorname{argmin}_{\mathbf{v} \in \mathcal{C}_{0}} \mathcal{P}_{0}\left(\mathbf{v}, \alpha_{0}\right) ; \boldsymbol{\sigma}_{s}$ denotes the real stress field at time $s$ and is given by (3), $\dot{\mathbf{U}}_{s}$ is the rate of a given (but arbitrarily chosen) admissible displacement field at time $s$ and $\dot{\mathcal{W}}_{s}^{e}$ denotes the linear form associated with the rate of the prescribed volume or surface forces at time $s$.

Remark 1 Our presentation will be essentially informal and we will assume that the fields are sufficiently smooth so that all the calculations make sense. A precise statement of the functional spaces which are needed to justify all steps is a very difficult task and remains far from the scope of the present paper. A natural requirement is that the total energy remains finite at each time. Accordingly, the damage field must belong to $L^{\infty}(\Omega) \cap H^{1}(\Omega)$. The question is more delicate for the displacement field because of the loss of stiffness on the crack. It will be partially treated when one will study the singularity of the fields at the neighborhood of the crack tip, see appendix A.

2.2 Preliminary results and fundamental assumptions

One immediately deduces that the stability condition (10) is satisfied only if, at each time, the body is at equilibrium and the damage criterion is satisfied. Specifically, they respectively read in a variational form as

$$
\begin{gathered}
\int_{\Omega} \boldsymbol{\sigma}_{t} \cdot \boldsymbol{\varepsilon}\left(\mathbf{v}-\mathbf{u}_{t}\right) \mathrm{d} x=\mathcal{W}_{t}^{e}\left(\mathbf{v}-\mathbf{u}_{t}\right), \quad \forall \mathbf{v} \in \mathcal{C}_{t}, \\
\int_{\Omega}\left(-\mathrm{Y}_{t} \cdot\left(\beta-\alpha_{t}\right)+\mathbf{q}_{t} \cdot \nabla\left(\beta-\alpha_{t}\right)\right) \mathrm{d} x \geq 0, \quad \forall \beta \in \mathcal{D}: \beta \geq \alpha_{t},
\end{gathered}
$$

where $\boldsymbol{\sigma}_{t}, \mathbf{Y}_{t}$ and $\mathbf{q}_{t}$ denote respectively the stress tensor, the energy release rate density and damage flux vector which are given in terms of the current state by the constitutive relations (3). These two conditions can be seen as the first order stability conditions. They are necessary but not always sufficient in order for (10) to hold Pham and Marigo (2010a,b).

From the numerical point of view Bourdin $(2007 \mathrm{a}, \mathrm{b})$, one uses an algorithm of alternate minimization in order to find the state of the body at each time step. That consists in constructing at time step $t_{i}$ a sequence $\left(\mathbf{u}_{t_{i}}^{n} \alpha_{t_{i}}^{n}\right)$, $n \in \mathbb{N}$, such that

$$
\mathbf{u}_{t_{i}}^{n}=\underset{\mathbf{v} \in \mathcal{C}_{t_{i}}}{\operatorname{argmin}} \mathcal{P}_{t_{i}}\left(\mathbf{v}, \alpha_{t_{i}}^{n}\right), \quad \alpha_{t_{i}}^{n+1}=\underset{\beta \in \mathcal{D}: \beta \geq \alpha_{t_{i-1}}}{\operatorname{argmin}} \mathcal{P}_{t_{i}}\left(\mathbf{u}_{t_{i}}, \beta\right),
$$


(with an initial condition, for example $\alpha_{t_{i}}^{0}=\alpha_{t_{i-1}}$ ). This algorithm is an algorithm of descent of the total energy which in general converges. In such a case it converges to a state $\left(\mathbf{u}_{t_{i}}, \alpha_{t_{i}}\right)$ which satisfies the first order stability conditions (12)-(13) at time step $t_{i}$.

Example 1 A typical example of numerical results which can be obtained by this type of damage models with the alternate minimization algorithm is illustrated in Figure 1. It corresponds to a thermal shock: the rectangular body is initially at a uniform temperature $T_{0}$ and the upper side $\left(x_{2}=0\right)$ is submitted to a lower temperature $T_{1}$ from time 0 . In the simulations the energy functions $\psi$ and $\mathrm{w}$ are taken as

$$
\psi(\varepsilon, \alpha)=\frac{1}{2}(1-\alpha)^{2} \mathrm{~A}_{0}\left(\varepsilon-\mathrm{a}_{0}\left(T-T_{0}\right) \mathbf{I}\right) \cdot\left(\varepsilon-\mathrm{a}_{0}\left(T-T_{0}\right) \mathbf{I}\right), \quad \mathrm{w}(\alpha)=\mathrm{w}_{1} \alpha,
$$

where $\mathrm{A}_{0}$ and $\mathrm{a}_{0}$ are respectively the stiffness tensor and the coefficient of thermal expansion of the (isotropic) material. In (14), $T$ denotes the current temperature of the considered material point. This temperature is obtained by solving the non stationary heat equation where one neglects the influence of the damage field. Specifically, the transient temperature field reads as

$$
T(\mathbf{x}, t)=T_{0}+\left(T_{1}-T_{0}\right) \operatorname{erfc}\left(\frac{x_{2}}{2 \sqrt{\kappa t}}\right)
$$

where erfc it the complementary error function, i.e. erfc $(x)=\frac{2}{\sqrt{\pi}} \int_{x}^{\infty} e^{-s^{2}} \mathrm{~d} s$, and $\kappa$ is a constant which characterizes the thermal conductivity of the material.

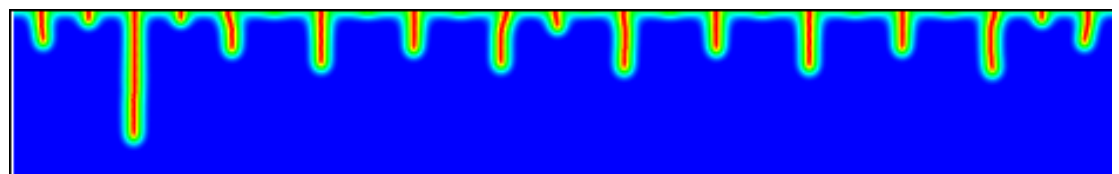

Fig. 1 Damage distribution obtained at a "large" time with an alternate minimization algorithm Bourdin (2007a,b); Bourdin et al. (2008) in the case of a thermal shock. The blue zones correspond to an undamaged point $(\alpha=0)$, the midline of the red zones to a totally damaged point $(\alpha=1)$.

The main stages of the damage evolution are the following ones: ( $i)$ the onset of damage occurs at $t=0$ at the upper side where the thermal shock happens, under the condition that $T_{0}-T_{1}$ is sufficiently large; (ii) for small $t$, the damage penetrates progressively inside the body but remains homogeneous in the horizontal direction; (iii) at some critical time, this homogeneous solution becomes unstable; (iv) then damage localizes in a set of periodically distributed zones; $(v)$ the damage parameter grows until 1 in the midline of these zones whose width remains of the order of $\eta$; (vi) the length of all the damage bands grows; (vii) finally, some damage bands stop to propagate whereas the other ones continue.

We are essentially interested here by the stages (vi) and (vii) of the evolution. In other words we assume that the damage bands have been previously created so that their width is of the order of $\eta$ whereas their length is much greater than $\eta$ such that the core of these bands is totally damaged. We will study the propagation of such a crack band by assuming that the path is given (here, the crack bands propagate along straight lines), but we do not try to explain why they are periodically distributed. This leads to the following:

Hypothesis 2 (The structuration in crack bands) Let $\Gamma_{t}$ be the set of totally damaged points and let $\gamma_{t}$ be the set of damage points at time $t$, i.e.

$$
\Gamma_{t}=\{\mathbf{x} \in \Omega: \alpha(\mathbf{x}, t)=1\} \quad \subset \quad \gamma_{t}=\{\mathbf{x} \in \Omega: 0<\alpha(\mathbf{x}, t) \leq 1\} .
$$

The former set will be called the crack whereas the latter will be called the crack band. We assume that 
1. $\Gamma_{t}$ is a smooth curve whose end $\mathbf{P}_{t}$ (the crack tip) is moving such that the length of the curve be a smooth increasing function of time, say $\ell_{t}$;

2. We consider the stage of the evolution such that $\ell_{t}$ is much larger that the material length $\eta$;

3. $\gamma_{t}$ is contained in a thin band included in $\Omega$, whose length differs from $\ell_{t}$ by a term of the order of $\eta$ and whose cross-section has a width of the order of $\eta$;

4. The damage evolution is smooth both in time and space.

Note that we will only consider the case with one crack band, but since our analysis is essentially local the procedure could easily be extended to the case of a family of crack bands like in the example of the thermal shock.

2.3 Some consequences of the first order stability conditions and of the energy balance

By standard arguments of the calculus of variations and by virtue of the hypothesis of regularity of the fields, one easily deduces from the first order stability conditions (12)-(13) the following

Proposition 1 The first order stability conditions are satisfied if and only if the following local conditions hold:

$$
\begin{aligned}
& \operatorname{div} \boldsymbol{\sigma}_{t}+\mathbf{f}_{t}=\mathbf{0} \text { in } \Omega \backslash \Gamma_{t}, \boldsymbol{\sigma}_{t} \mathbf{n}=\mathbf{F}_{t} \text { on } \partial_{N} \Omega, \quad \boldsymbol{\sigma}_{t} \mathbf{n}=\mathbf{0} \text { on } \Gamma_{t}, \\
& \mathbf{Y}_{t}+\operatorname{div} \mathbf{q}_{t} \leq 0 \text { in } \Omega \backslash \Gamma_{t}, \mathbf{q}_{t} \cdot \mathbf{n} \geq 0 \text { on } \partial \Omega .
\end{aligned}
$$

Note that the two bulk conditions hold only in the uncracked part of the body.

It remains to use the energy balance (11). Owing to the smoothness assumption on the time evolution, one can take the derivative of (11) with respect to $t$ which leads to

$$
\begin{aligned}
0 & =\frac{d}{d t} \mathcal{P}_{t}\left(\mathbf{u}_{t}, \alpha_{t}\right)-\int_{\Omega \backslash \Gamma_{t}} \boldsymbol{\sigma}_{t} \cdot \varepsilon\left(\dot{\mathbf{U}}_{t}\right) \mathrm{d} x+\mathcal{W}_{t}^{e}\left(\dot{\mathbf{U}}_{t}\right)+\dot{\mathcal{W}}_{t}^{e}\left(\mathbf{u}_{t}\right) \\
& =\frac{d}{d t}\left(\int_{\Omega \backslash \Gamma_{t}} W_{t} \mathrm{~d} x\right)-\int_{\Omega \backslash \Gamma_{t}} \boldsymbol{\sigma}_{t} \cdot \varepsilon\left(\dot{\mathbf{U}}_{t}\right) \mathrm{d} x-\mathcal{W}_{t}^{e}\left(\dot{\mathbf{u}}_{t}-\dot{\mathbf{U}}_{t}\right),
\end{aligned}
$$

where $W_{t}=W\left(\varepsilon\left(\mathbf{u}_{t}\right), \alpha_{t}, \nabla \alpha_{t}\right)$. The main difficulty is to evaluate the rate of the bulk energy near the tip of the crack when the crack tip moves because of the possible presence of singularities. Accordingly, we partition $\Omega$ to isolate the (moving) tip of the crack. Let $\mathbb{B}_{r}(t)$ be the ball of radius $r$ centered at $\mathbf{P}_{t}$, let $\mathbb{C}_{r}(t)$ be its boundary (a circle of radius $r$ ) and let $\Omega_{r}(t)$ be the uncracked part of the body outside the ball $\mathbb{B}_{r}(t): \Omega_{r}(t)=\Omega \backslash\left(\Gamma_{t} \cup \mathbb{B}_{r}(t)\right)$. The unit tangent vector to $\Gamma_{t}$ at $\mathbf{P}_{t}$ is denoted $\boldsymbol{\tau}_{t}$ (Fig. 2). Let us evaluate the derivative with respect to $t$ of the bulk energy included in $\Omega_{r}(t)$. Using classical results for the derivative of integrals over time dependent domains Germain (1973) and by virtue of the regularity assumption, one gets

$$
\frac{d}{d t}\left(\int_{\Omega_{r}(t)} W_{t} \mathrm{~d} x\right)=\int_{\Omega_{r}(t)}\left(\boldsymbol{\sigma}_{t} \cdot \boldsymbol{\varepsilon}\left(\dot{\mathbf{u}}_{t}\right)-\mathbf{Y}_{t} \cdot \dot{\alpha}_{t}+\mathbf{q}_{t} \cdot \nabla \dot{\alpha}_{t}\right) \mathrm{d} x-\dot{\ell}_{t} \int_{\mathbb{C}_{r}(t)} W_{t} \boldsymbol{\tau}_{t} \cdot \mathbf{n} \mathrm{d} s,
$$

where $\mathbf{n}$ denotes the outer unit normal to $\mathbb{B}_{r}(t)$. Inserting (19) into (18) leads to

$$
\begin{aligned}
0= & \int_{\Omega_{r}(t)}\left(\boldsymbol{\sigma}_{t} \cdot \boldsymbol{\varepsilon}\left(\dot{\mathbf{u}}_{t}-\dot{\mathbf{U}}_{t}\right)-\mathbf{f}_{t} \cdot\left(\dot{\mathbf{u}}_{t}-\dot{\mathbf{U}}_{t}\right)-\mathbf{Y}_{t} \cdot \dot{\alpha}_{t}+\mathbf{q}_{t} \cdot \nabla \dot{\alpha}_{t}\right) \mathrm{d} x-\int_{\partial_{N} \Omega} \mathbf{F}_{t} \cdot\left(\dot{\mathbf{u}}_{t}-\dot{\mathbf{U}}_{t}\right) \mathrm{d} s \\
& -\dot{\ell}_{t} \int_{\mathbb{C}_{r}(t)} W_{t} \mathbf{n} \cdot \boldsymbol{\tau}_{t} \mathrm{~d} s+\frac{d}{d t}\left(\int_{\mathbb{B}_{r}(t)} W_{t} \mathrm{~d} x\right)-\int_{\mathbb{B}_{r}(t)}\left(\boldsymbol{\sigma}_{t} \cdot \varepsilon\left(\dot{\mathbf{U}}_{t}\right)+\mathbf{f}_{t} \cdot\left(\dot{\mathbf{u}}_{t}-\dot{\mathbf{U}}_{t}\right)\right) \mathrm{d} x .
\end{aligned}
$$




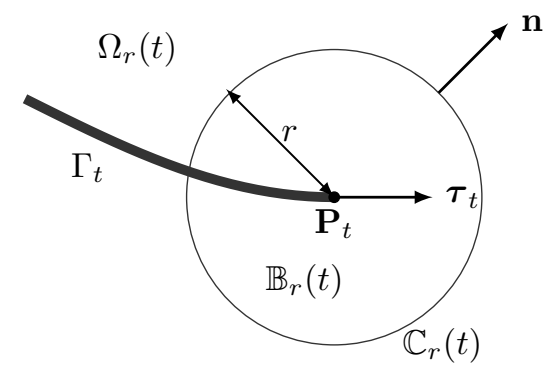

Fig. 2 The crack tip moving with the speed $\dot{\ell}_{t}$. The contour $\mathbb{C}_{r}(t)$ translates with the crack tip.

Integrating by parts the gradient terms in the integral over $\Omega_{r}(t)$ in (20) and using the equilibrium equations of (17) allow to simplify the energy balance which can read now

$$
\begin{aligned}
0= & \int_{\Omega_{r}(t)}\left(\mathbf{Y}_{t}+\operatorname{div} \mathbf{q}_{t}\right) \cdot \dot{\alpha}_{t} \mathrm{~d} x+\int_{\mathbb{C}_{r}(t)}\left(W_{t} \mathbf{n} \cdot \boldsymbol{\tau}_{t} \dot{\ell}_{t}+\boldsymbol{\sigma}_{t} \mathbf{n} \cdot \dot{\mathbf{u}}_{t}+\mathbf{q}_{t} \cdot \mathbf{n} \dot{\alpha}_{t}\right) \mathrm{d} s \\
& -\frac{d}{d t}\left(\int_{\mathbb{B}_{r}(t)} W_{t} \mathrm{~d} x\right)+\int_{\mathbb{B}_{r}(t)}\left(\boldsymbol{\sigma}_{t} \cdot \varepsilon\left(\dot{\mathbf{U}}_{t}\right)+\mathbf{f}_{t} \cdot\left(\dot{\mathbf{u}}_{t}-\dot{\mathbf{U}}_{t}\right)\right) \mathrm{d} x .
\end{aligned}
$$

To conclude we must pass to the limit when $r$ goes to 0 . Under suitable assumptions on the behavior of the fields near the crack tip, we will show that the integrals over $\mathbb{B}_{r}(t)$ do not give a contribution to the limit because their integrand cannot be sufficiently singular, see appendix A. On the other hand, the integral over $\mathbb{C}_{r}(t)$ can give a non null limit. Its study will be made in the next section and in appendix A. For that purpose, it is more convenient to make a change of coordinates by taking the tip of the crack $\mathbf{P}_{t}$ as the (moving) origin of the coordinates and $\left(\tilde{x}_{1}, \tilde{x}_{2}\right)$ as the new cartesian coordinates with $\tilde{x}_{1}=\left(\mathbf{x}-\mathbf{P}_{t}\right) \cdot \boldsymbol{\tau}_{t}$. Specifically, one sets $\tilde{\mathbf{x}}=\left(\mathbf{x}-\mathbf{P}_{t}\right)$ and any field $f$ is transformed into $\tilde{f}$ so that $\tilde{f}(\tilde{\mathbf{x}}, t)=f\left(\mathbf{P}_{t}+\tilde{\mathbf{x}}, t\right)$. By the chain rule one gets

$$
\dot{f}(\mathbf{x}, t)=\frac{\partial \tilde{f}}{\partial t}(\tilde{\mathbf{x}}, t)-\dot{\ell}_{t} \frac{\partial \tilde{f}}{\partial \tilde{x}_{1}}(\tilde{\mathbf{x}}, t)
$$

Inserting this change into the integral over $\mathbb{C}_{r}(t)$ which becomes an integral over $\tilde{\mathbb{C}}_{r},(21)$ can read as

$$
0=\int_{\Omega_{r}(t)}\left(\mathrm{Y}_{t}+\operatorname{div} \mathbf{q}_{t}\right) \cdot \dot{\alpha}_{t} \mathrm{~d} x+\mathrm{J}_{r}(t) \dot{\ell}_{t}+\mathrm{I}_{r}(t)
$$

with

$$
\mathrm{J}_{r}(t)=\int_{\tilde{\mathbb{C}}_{r}}\left(\tilde{W}_{t} \tilde{n}_{1}-\tilde{\boldsymbol{\sigma}}_{t} \tilde{\mathbf{n}} \cdot \frac{\partial \tilde{\mathbf{u}}_{t}}{\partial \tilde{x}_{1}}-\tilde{\mathbf{q}}_{t} \cdot \tilde{\mathbf{n}} \frac{\partial \tilde{\alpha}_{t}}{\partial \tilde{x}_{1}}\right) \mathrm{d} \tilde{s}
$$

and

$$
\mathrm{I}_{r}(t)=\int_{\tilde{\mathbb{C}}_{r}}\left(\tilde{\boldsymbol{\sigma}}_{t} \tilde{\mathbf{n}} \cdot \frac{\partial \tilde{\mathbf{u}}_{t}}{\partial t}+\tilde{\mathbf{q}} t \cdot \tilde{\mathbf{n}} \frac{\partial \tilde{\alpha}_{t}}{\partial t}\right) \mathrm{d} \tilde{s}-\frac{d}{d t}\left(\int_{\mathbb{B}_{r}(t)} W_{t} \mathrm{~d} x\right)+\int_{\mathbb{B}_{r}(t)}\left(\boldsymbol{\sigma}_{t} \cdot \boldsymbol{\varepsilon}\left(\dot{\mathbf{U}}_{t}\right)+\mathbf{f}_{t} \cdot\left(\dot{\mathbf{u}}_{t}-\dot{\mathbf{U}}_{t}\right)\right) \mathrm{d} x
$$

We are now in a position to state the second fundamental result of the paper. However, for technical reasons, the proof is only established in the particular case of Example 1 and with an a priori assumption on the form of the fields near the crack tip. 
Proposition 2 In the case of the model of Example 1, i.e. $\mathrm{A}(\alpha)=(1-\alpha)^{2} \mathrm{~A}_{0}$ and $\mathrm{w}(\alpha)=\mathrm{w}_{1} \alpha$, we assume that the most singular part of the damage and displacement fields in the neighborhood of the crack tip can read as

$$
1-\alpha(r, \theta)=r^{p} V(\theta)+\cdots, \quad \mathbf{u}(r, \theta)=r^{q}\left(U_{r}(\theta) e_{r}+U_{\theta}(\theta) e_{\theta}\right)+\cdots,
$$

where $p$ and $q$ are real numbers such that the total energy be finite, i.e. $p>0$ and $p+q>0$. Under these conditions, one first proves that

$$
\lim _{r \rightarrow 0} \mathrm{I}_{r}(t)=0, \quad \lim _{r \rightarrow 0} \mathrm{~J}_{r}(t)=\mathrm{J}_{0}(t) \leq 0 .
$$

Then, from the first order stability conditions and the global energy balance, one deduces that

$$
\left(\mathrm{Y}_{t}+\operatorname{div} \mathbf{q}_{t}\right) \cdot \dot{\alpha}_{t}=0 \text { in } \Omega \backslash \Gamma_{t}, \quad \mathrm{~J}_{0}(t) \dot{\ell}_{t}=0,
$$

which can be seen as the local energy balances.

Proof The proof of the property (24) is given in appendix A. Accordingly, since $\lim _{r \rightarrow 0} I_{r}(t)=0$, passing to the limit when $r$ goes to 0 in (22) gives

$$
0=\int_{\Omega \backslash \Gamma_{t}}\left(\mathrm{Y}_{t}+\operatorname{div} \mathbf{q}_{t}\right) \cdot \dot{\alpha}_{t} \mathrm{~d} x+\mathrm{J}_{0}(t) \dot{\ell}_{t} .
$$

Since $\mathbf{Y}_{t}+\operatorname{div} \mathbf{q}_{t} \leq 0$ by virtue of the first order stability conditions and since $\dot{\alpha}_{t} \geq 0$ by virtue of the irreversibility condition, the integral over $\Omega_{r}(t)$ in $(22)$ is non positive. Since $J_{0}(t) \leq 0$ and since $\dot{\ell}_{t} \geq 0$ by virtue of the irreversibility condition, $\mathrm{J}_{0}(t) \dot{\ell}_{t} \leq 0$. Therefore, each term in (26) is non positive while their sum must be zero, hence each term vanishes which is precisely $(25)$.

Hypothesis 3 We assume that Proposition 2 can be extended to the general class of strongly brittle materials considered in Section 2. Hence, the damage criterion and the local energy balance read

$$
\begin{gathered}
\frac{1}{2} \mathrm{~S}^{\prime}\left(\alpha_{t}\right) \boldsymbol{\sigma}_{t} \cdot \boldsymbol{\sigma}_{t}-\mathrm{w}^{\prime}\left(\alpha_{t}\right)+\mathrm{w}_{1} \eta^{2} \Delta \alpha_{t} \leq 0 \quad \text { in } \quad \Omega \backslash \Gamma_{t}, \\
\left(\frac{1}{2} \mathrm{~S}^{\prime}\left(\alpha_{t}\right) \boldsymbol{\sigma}_{t} \cdot \boldsymbol{\sigma}_{t}-\mathrm{w}^{\prime}\left(\alpha_{t}\right)+\mathrm{w}_{1} \eta^{2} \Delta \alpha_{t}\right) \dot{\alpha}_{t}=0 \quad \text { in } \quad \Omega \backslash \Gamma_{t} .
\end{gathered}
$$

\section{Separation of scale and Griffith's law}

All the results of the previous section have been obtained without using the assumption that the internal length of the material $\eta$ is small by comparison with the length of the crack and the dimensions of the body. In particular the property that $\mathrm{J}_{0}(t) \dot{\ell}_{t}=0$ does not require such an assumption. On the other hand, the link with Griffith's law can only be made in this asymptotic context. Therefore, we adopt this assumption henceforth. Moreover, we still consider the family of strongly brittle materials introduced in Section 2. To rigorously make a separation of scale requires the use of asymptotic methods like matched asymptotic expansions Lagerstrom (1988). The comprehensive description of such a procedure is outside the scope of this paper. We merely present here its great lines. Throughout this section, $\eta$ is considered as a small parameter and the dependence on it of any quantity is indicated by a superscript like $\mathbf{u}^{\eta}$ for the displacement field. On the contrary, the explicit time dependence is now removed. 
1. The damage evolution problem can be decomposed into three problems: (i) the outer problem which gives the behavior of the fields far enough from the crack band and the crack tip; (ii) the crack band problem which gives the behavior of the fields in or near the crack band but far enough from the crack tip; (iii) the crack tip problem which gives the behavior of the fields in the neighborhood of the crack tip.

2. The order of magnitude of the fields with respect to the small parameter $\eta$ depends first on the order of magnitude of the loading and then on the zone where they are evaluated. It turns out that it is sufficient to prescribe a loading $\left(\mathbf{f}^{\eta}, \mathbf{F}^{\eta}, \mathbf{U}^{\eta}\right.$ or $\left.\varepsilon^{0 \eta}\right)$ with a magnitude the order of $\sqrt{\eta}$ for propagating the crack. This is essentially due to the stress concentration which is automatically induced by the presence of the tip of the crack and to the fact that the dissipated energy for creating the crack band is of the order of $\eta$. We assume that the amplitude of the loading is of the order of $\sqrt{\eta}$ :

$$
\mathbf{f}^{\eta}=\sqrt{\eta} \mathbf{f}, \quad \mathbf{F}^{\eta}=\sqrt{\eta} \mathbf{F}, \quad \mathbf{U}^{\eta}=\sqrt{\eta} \mathbf{U}, \quad \varepsilon^{0 \eta}=\sqrt{\eta} \varepsilon^{0} .
$$

3.1 The outer problem and definition of Griffith's energy release rate

At a macroscale the thin process zone around the crack can be neglected and the associated outer problem is a purely elastic problem posed on the cracked domain $\Omega \backslash \Gamma$. Its solution depends linearly on the loading parameters. Since the magnitude of the loading is of the order of $\sqrt{\eta}$, the real displacement and stress fields can read

$$
\mathbf{u}^{\eta}=\sqrt{\eta} \mathbf{u}+\cdots, \quad \boldsymbol{\sigma}^{\eta}=\sqrt{\eta} \boldsymbol{\sigma}+\cdots,
$$

where $\mathbf{u}$ and $\boldsymbol{\sigma}$ satisfy

$$
\begin{array}{r}
\operatorname{div} \boldsymbol{\sigma}+\mathbf{f}=0, \quad \boldsymbol{\sigma}=\mathrm{A}_{0}\left(\varepsilon(\mathbf{u})-\boldsymbol{\varepsilon}^{0}\right) \quad \text { in } \quad \Omega \backslash \Gamma, \\
\boldsymbol{\sigma} \mathbf{n}=\mathbf{F} \text { on } \partial_{N} \Omega, \quad \boldsymbol{\sigma} \mathbf{n}=\mathbf{0} \text { on } \Gamma, \quad \mathbf{u}=\mathbf{U} \text { on } \partial_{D} \Omega .
\end{array}
$$

The fields $\mathbf{u}$ and $\boldsymbol{\sigma}$ are singular near the tip of the crack (but of course these fields are not a good approximation of the real fields in that zone). In the case of an isotropic material, the singular part of $\sigma$ is

$$
\boldsymbol{\sigma}(\mathbf{x})=\frac{\bar{K}_{I}}{\sqrt{r}} \boldsymbol{\Sigma}_{I}(\theta)+\frac{\bar{K}_{I I}}{\sqrt{r}} \boldsymbol{\Sigma}_{I I}(\theta)+\cdots,
$$

where $\bar{K}_{I}, \bar{K}_{I I}$ are the (rescaled) stress intensity factors and $\boldsymbol{\Sigma}_{I}, \boldsymbol{\Sigma}_{I I}$ the usual angular functions corresponding to the opening and sliding modes Leblond (2000).

We can also define in this fictitious elastic problem the potential energy release rate $G^{\eta}$ which is related to the stress intensity factors by Irwin's formula Leblond (2000). By linearity, one gets

$$
G^{\eta}=\eta \bar{G}, \quad \bar{G}=\frac{1-\nu_{0}^{2}}{E_{0}}\left(\bar{K}_{I}^{2}+\bar{K}_{I I}^{2}\right)
$$

where $E_{0}$ and $\nu_{0}$ are the Young modulus and the Poisson ratio of the sound material. Note that $G^{\eta}$ is of the order of $\eta$ by virtue of the assumption on the loading magnitude. In the same manner the real stress intensity factors $K_{I, I I}^{\eta}$ are of order $\sqrt{\eta}: K_{I, I I}^{\eta}=\sqrt{\eta} \bar{K}_{I, I I}$. 
3.2 The damage field inside the crack band and definition of $G_{C}$

In the crack band or in its neighborhood but far enough from the crack tip, the displacement and stress fields are matched to those of the outer problem. It follows in particular that the stresses are still of order $\sqrt{\eta}$. Let $s$ be the arclength and $s \mapsto \chi(s)$ be a parameterization of $\Gamma$. A point $\mathbf{x}$ in the crack band is represented by the system of curvilinear coordinates $(s, \zeta)$ such that $\mathbf{x}=\boldsymbol{\chi}(s)+\zeta \eta \boldsymbol{\nu}(s)$ where $\boldsymbol{\nu}(s)$ is unit normal vector to $\Gamma$ at $\boldsymbol{\chi}(s)$ (Fig. 3). The damage field $\alpha^{\eta}$ inside the crack band or in its neighborhood is assumed to be of the form

$$
\alpha^{\eta}(\mathbf{x})=\alpha_{*}(s, \zeta)+\text { higher order terms } \quad \text { with } \quad \alpha_{*}(s, 0)=1 \quad \text { and } \quad \alpha_{*}(s, \zeta)=0 \text { for }|\zeta| \geq \bar{D}(s) .
$$

Hence $2 \bar{D}(s) \eta$ represents the width of the crack band at $s$. Let us remark that $\frac{1}{2} \mathrm{~S}^{\prime}\left(\alpha^{\eta}\right) \boldsymbol{\sigma}^{\eta} \cdot \boldsymbol{\sigma}^{\eta}$ is of the order of $\eta$ whereas $\mathrm{w}^{\prime}\left(\alpha^{\eta}\right)$ and $\operatorname{div} \mathbf{q}^{\eta}=\mathrm{w}_{1} \eta^{2} \Delta \alpha^{\eta}$ are of the order of 1 . Since the gradient of $\alpha^{\eta}$ in the tangential direction is negligible by comparison with its gradient in the normal direction, the damage criterion (27) inside or in the neighborhood of the crack band can read at the first order as $w^{\prime}\left(\alpha_{*}\right)-w_{1} \frac{\partial^{2} \alpha_{*}}{\partial \zeta^{2}} \leq 0$. Moreover, by virtue of (28) this inequality is an equality when the damage is evolving. Accordingly, we can consider that the equality holds once the band is created and then

$$
\mathrm{w}^{\prime}\left(\alpha_{*}(\zeta)\right)-\mathrm{w}_{1} \frac{\partial^{2} \alpha_{*}}{\partial \zeta^{2}}(\zeta)=0 \quad \text { for } \quad|\zeta| \leq \bar{D}(s) .
$$

This autonomous second order ordinary differential equation admits a first integral. Indeed, multiplying it by $\partial \alpha_{*} / \partial \zeta$, it appears that $\mathrm{w}\left(\alpha_{*}\right)-\frac{1}{2} \mathrm{w}_{1}\left(\frac{\partial \alpha_{*}}{\partial \zeta}\right)^{2}$ is independent of $\zeta$. Using the boundary conditions at $\zeta= \pm \bar{D}(s)$, one gets that this constant is 0 and hence

$$
\sqrt{\frac{\mathrm{w}_{1}}{2 \mathrm{w}\left(\alpha_{*}\right)}} \frac{\partial \alpha_{*}}{\partial \zeta}=\operatorname{sign}(\zeta)
$$

Using the boundary condition at $\zeta=0$ one finally obtains

$$
\bar{D}=\int_{0}^{1} \sqrt{\frac{\mathrm{w}_{1}}{2 \mathrm{w}(\beta)}} \mathrm{d} \beta, \quad|\zeta|=\int_{\alpha_{*}(\zeta)}^{1} \sqrt{\frac{\mathrm{w}_{1}}{2 \mathrm{w}(\beta)}} \mathrm{d} \beta,
$$

$\alpha_{*}(\zeta)$ being given implicitly. It turns out that $\bar{D}$ and $\alpha_{*}$ are independent of $s$, which means that the damage profile is the same all along the crack band (except at its ends).

Let us calculate now the energy by unit length dissipated during the creation of the crack band. It is given by the integral over the crack band cross-section of $\mathrm{w}\left(\alpha^{\eta}\right)+\frac{1}{2} \mathrm{w}_{1} \eta^{2} \nabla \alpha^{\eta} \cdot \nabla \alpha^{\eta}$. Both terms in that sum are of the order of $\eta$ and by virtue of (31) give the same contribution to the integral. Denoting by $G_{c}^{\eta}$ the leading term of the dissipated energy, after an easy calculation one obtains

$$
G_{c}^{\eta}=\eta \bar{G}_{c}, \quad \bar{G}_{c}=2 \sqrt{2} \int_{0}^{1} \sqrt{\mathrm{w}_{1} \mathrm{w}(\beta)} \mathrm{d} \beta .
$$

Note that $G_{c}^{\eta}$ is of the order of $\eta$, intrinsic to the material and independent of the crack path and how the crack band was created. It will play the role of the surface energy density in Griffith's law.

Example 2 In the case of the constitutive law of Example 1 where $\mathrm{A}(\alpha)=(1-\alpha)^{2} \mathrm{~A}_{0}$ and $\mathrm{w}(\alpha)=\mathrm{w}_{1} \alpha$, one gets $\bar{D}=\sqrt{2}$ (hence the width of the crack band is $2 \sqrt{2} \eta$ ), $G_{c}^{\eta}=4 \sqrt{2} \mathrm{w}_{1} \eta / 3$ whereas the critical stress $\sigma_{c}$ in a uniaxial tensile test is independent of $\eta$ and given by $\sigma_{c}=\sqrt{\mathrm{w}_{1} E_{0}}$. Moreover the damage profile in the crack band is a parabola: $\alpha_{*}(\zeta)=(1-|\zeta| / \sqrt{2})^{2}$. 

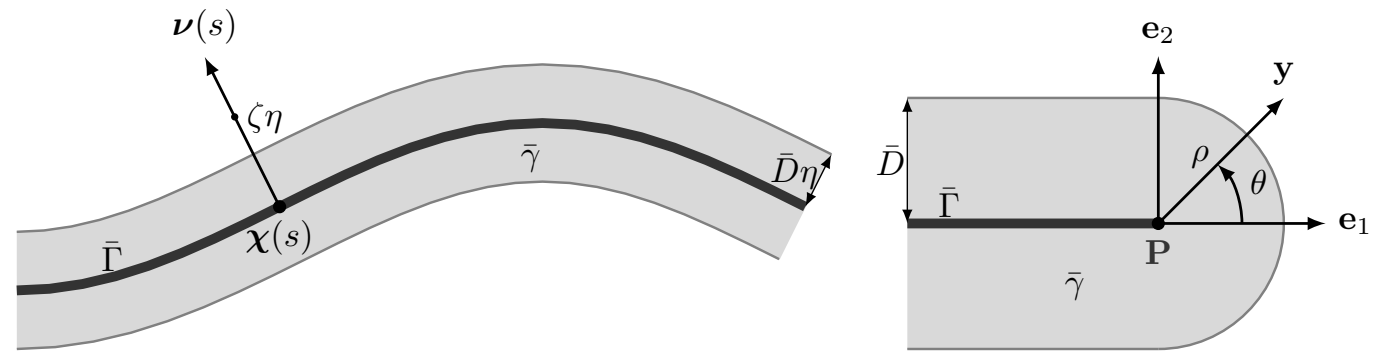

Fig. 3 The damage band

Fig. 4 The crack tip with the damage zone

3.3 The damage problem near the tip of the crack

Near the tip of the crack, $\eta$ is the natural length scale. On that account let us rescale the system of coordinates in the neighborhood of the crack tip $\mathbf{P}=\boldsymbol{\chi}(\ell)$ by setting

$$
\mathbf{y}=\frac{\mathbf{x}-\mathbf{P}}{\eta}=y_{1} \mathbf{e}_{1}+y_{2} \mathbf{e}_{2}, \quad \rho=\|\mathbf{y}\|=\frac{r}{\eta}
$$

This crack tip problem is posed in the entire plane $\mathbb{R}^{2}$, the crack corresponding to the half-line $\bar{\Gamma}=(-\infty, 0) \times\{0\}$ and the crack band to $\bar{\gamma}$ (Fig. 4). One searches for the displacement, stress and damage fields under the form

$$
\mathbf{u}^{\eta}(\mathbf{x})=\sqrt{\eta} \mathbf{u}(\mathbf{P})+\eta \overline{\mathbf{u}}(\mathbf{y})+\cdots, \quad \boldsymbol{\sigma}^{\eta}(\mathbf{x})=\overline{\boldsymbol{\sigma}}(\mathbf{y})+\cdots, \quad \alpha^{\eta}(\mathbf{x})=\bar{\alpha}(\mathbf{y})+\cdots,
$$

where $\mathbf{u}(\mathbf{P})$ represents the displacement of the crack tip given by the outer problem. By virtue of a stress concentration effect due to the presence of the crack tip, the stresses are of the order of 1 in this region while they are of the order of $\sqrt{\eta}$ in the outer domain. Therefore, by matching the two expansions one gets the following behavior of $\overline{\boldsymbol{\sigma}}$ at infinity:

$$
\lim _{\rho \rightarrow \infty}\left(\overline{\boldsymbol{\sigma}}(\mathbf{y})-\frac{\bar{K}_{I}}{\sqrt{\rho}} \boldsymbol{\Sigma}_{I}(\theta)-\frac{\bar{K}_{I I}}{\sqrt{\rho}} \boldsymbol{\Sigma}_{I I}(\theta)\right)=\mathbf{0}
$$

and hence the rescaled stress intensity factors $\bar{K}_{I}$ and $\bar{K}_{I I}$ given by the outer problem will play the role of the time-dependent loading parameters for the crack tip problem. The equilibrium equations, the stress-strain relations and the boundary conditions read

$$
\operatorname{div} \overline{\boldsymbol{\sigma}}=\mathbf{0} \quad \text { and } \quad \overline{\boldsymbol{\sigma}}=\mathrm{A}(\bar{\alpha}) \boldsymbol{\varepsilon}(\overline{\mathbf{u}}) \quad \text { in } \quad \mathbb{R}^{2} \backslash \bar{\Gamma}, \quad \overline{\boldsymbol{\sigma}} \mathbf{e}_{2}=\mathbf{0} \quad \text { and } \quad \bar{\alpha}=1 \quad \text { on } \quad \bar{\Gamma},
$$

where all the spatial derivatives are now taken with respect to $\mathbf{y}$. Note that the loadings $\mathbf{f}^{\eta}$ and $\varepsilon^{0 \eta}$ do not appear because they are of higher order. Since all terms in $Y^{\eta}+\operatorname{div} \mathbf{q}^{\eta}$ are of the order of 1 , the damage criterion (27) reads at the first order as

$$
\frac{1}{2} \mathrm{~S}^{\prime}(\bar{\alpha}) \overline{\boldsymbol{\sigma}} \cdot \overline{\boldsymbol{\sigma}}-\mathrm{w}^{\prime}(\bar{\alpha})+\mathrm{w}_{1} \Delta \bar{\alpha} \leq 0 \quad \text { in } \quad \mathbb{R}^{2} \backslash \bar{\Gamma} .
$$

From the chain rule, the rate of damage is of order of $1 / \eta$ and reads

$$
\dot{\alpha}^{\eta}(\mathbf{x})=-\frac{\dot{\ell}}{\eta} \frac{\partial \bar{\alpha}}{\partial y_{1}}(\mathbf{y})+\cdots .
$$

Accordingly, the local energy balance (28) reads at the first order

$$
\left(\frac{1}{2} \mathrm{~S}^{\prime}(\bar{\alpha}) \overline{\boldsymbol{\sigma}} \cdot \overline{\boldsymbol{\sigma}}-\mathrm{w}^{\prime}(\bar{\alpha})+\mathrm{w}_{1} \Delta \bar{\alpha}\right) \frac{\partial \bar{\alpha}}{\partial y_{1}} \dot{\ell}=0 \quad \text { in } \quad \mathbb{R}^{2} \backslash \bar{\Gamma} .
$$


The damage field expansion of the crack tip problem must be matched to the damage field expansions of the outer and crack band problems. Therefore $\bar{\alpha}$ must vanish at infinity except in the direction of the crack where it must correspond to the field $\alpha_{*}$ given by (31). Specifically, these matching conditions read

$$
\lim _{\|y\| \rightarrow \infty, y_{2} \neq 0} \bar{\alpha}(\mathbf{y})=0, \quad \lim _{y_{1} \rightarrow-\infty} \bar{\alpha}(\mathbf{y})=\alpha_{*}\left(y_{2}\right) .
$$

3.4 The generalized Rice integral and its properties

In the analysis of the global energy balance, the flux of energy $J_{r}(t)$ defined in (23) is a path integral which generalizes Rice's integral used in brittle fracture to calculate the potential energy release rate. This path integral will be studied for paths which are very close to the tip of the crack. Using the rescaled system of coordinates $\left(y_{1}, y_{2}\right)$, let us consider such a path $\overline{\mathcal{C}}$ whose ends are located on the lips of the crack and which circumvents the crack tip (Fig. 5). It appears that the associated path integral $J_{\overline{\mathcal{C}}}^{\eta}$ is of the order of $\eta, J_{\overline{\mathcal{C}}}^{\eta}=\eta \bar{J}_{\overline{\mathcal{C}}}+\cdots$ and $\bar{J}_{\overline{\mathcal{C}}}$ reads as

$$
\bar{J}_{\overline{\mathcal{C}}}=\int_{\overline{\mathcal{C}}}\left(\bar{W}(\varepsilon(\overline{\mathbf{u}}), \bar{\alpha}, \nabla \bar{\alpha}) n_{1}-\frac{\partial \bar{W}}{\partial \bar{\varepsilon}_{i j}}(\varepsilon(\overline{\mathbf{u}}), \bar{\alpha}, \nabla \bar{\alpha}) n_{j} \frac{\partial \bar{u}_{i}}{\partial y_{1}}-\frac{\partial \bar{W}}{\partial \bar{g}_{j}}(\varepsilon(\overline{\mathbf{u}}), \bar{\alpha}, \nabla \bar{\alpha}) n_{j} \frac{\partial \bar{\alpha}}{\partial y_{1}}\right) \mathrm{d} \bar{s},
$$

where $\bar{s}$ denotes the arclength of $\overline{\mathcal{C}}$. In (40) $\bar{W}$ represents the rescaled bulk energy density, i.e.

$$
\bar{W}(\bar{\varepsilon}, \bar{\alpha}, \overline{\mathbf{g}})=\frac{1}{2} \mathrm{~A}(\bar{\alpha}) \bar{\varepsilon} \cdot \bar{\varepsilon}+\mathrm{w}(\bar{\alpha})+\frac{1}{2} \mathrm{w}_{1} \overline{\mathbf{g}} \cdot \overline{\mathbf{g}}
$$

and one sets $\overline{\mathbf{q}}=\frac{\partial \bar{W}}{\partial \overline{\mathbf{g}}}=\mathrm{w}_{1} \overline{\mathbf{g}}$. One fundamental property of the path integral is its monotonicity with respect to

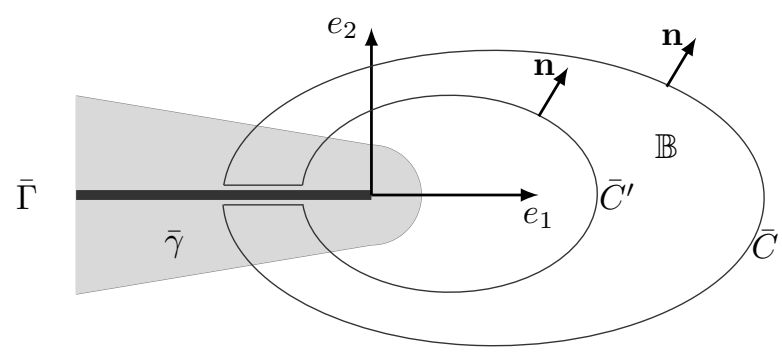

Fig. 5 Two ordered paths in the neighborhood of the crack tip.

the path. This requires to define the following partial order relation between the paths

Definition 1 One says that the path $\overline{\mathcal{C}}$ is greater than the path $\overline{\mathcal{C}}^{\prime}$, if $\overline{\mathcal{C}}^{\prime}$ is included in the domain delimited by the path $\overline{\mathcal{C}}$ and the crack $\bar{\Gamma}$.

Let us prove the first fundamental property of this path integral.

Proposition 3 When the crack propagates, i.e. when $\dot{\ell}>0$, then the path integral $\bar{J}_{\overline{\mathcal{C}}}$ is path independent. 
Proof Let us consider two paths $\overline{\mathcal{C}}$ and $\overline{\mathcal{C}}^{\prime}$ such that $\overline{\mathcal{C}} \geq \overline{\mathcal{C}}^{\prime}$ in the sense of Definition 1 (see also Fig 5 ). Let $\mathbb{B}$ be the domain delimited by theses two paths and the crack line. It is easy to check that

$$
\bar{J}_{\overline{\mathcal{C}}}-\bar{J}_{\overline{\mathcal{C}}^{\prime}}=\int_{\partial \mathbb{B}}\left(\bar{W} n_{1}-\bar{\sigma}_{i j} n_{j} \frac{\partial \bar{u}_{i}}{\partial y_{1}}-\bar{q}_{j} n_{j} \frac{\partial \bar{\alpha}}{\partial y_{1}}\right) \mathrm{d} \bar{s},
$$

because $n_{1}=0, \overline{\boldsymbol{\sigma}} \mathbf{n}=\mathbf{0}$ and $\partial \bar{\alpha} / \partial y_{1}=0$ on $\bar{\Gamma}$. By Green's formula one gets

$$
\begin{aligned}
\bar{J}_{\overline{\mathcal{C}}}-\bar{J}_{\overline{\mathcal{C}}^{\prime}} & =\int_{\mathbb{B}}\left(\frac{\partial \bar{W}}{\partial y_{1}}-\frac{\partial}{\partial y_{j}}\left(\bar{\sigma}_{i j} \frac{\partial \bar{u}_{i}}{\partial y_{1}}+\bar{q}_{j} \frac{\partial \bar{\alpha}}{\partial y_{1}}\right)\right) \mathrm{d} \bar{s} \\
& =\int_{\mathbb{B}}\left(\frac{\partial \bar{W}}{\partial \bar{\alpha}} \frac{\partial \bar{\alpha}}{\partial y_{1}}-\frac{\partial \bar{\sigma}_{i j}}{\partial y_{j}} \frac{\partial \bar{u}_{i}}{\partial y_{1}}-\operatorname{div} \bar{q} \frac{\partial \bar{\alpha}}{\partial y_{1}}\right) \mathrm{d} \bar{s}
\end{aligned}
$$

Using the equilibrium equation $\operatorname{div} \overline{\boldsymbol{\sigma}}=\mathbf{0}$ and the definitions of $\bar{W}$ leads to

$$
\bar{J}_{\overline{\mathcal{C}}}-\bar{J}_{\overline{\mathcal{C}}^{\prime}}=-\int_{\mathbb{B}}\left(\frac{1}{2} \mathrm{~S}^{\prime}(\bar{\alpha}) \overline{\boldsymbol{\sigma}} \cdot \overline{\boldsymbol{\sigma}}-\mathrm{w}^{\prime}(\bar{\alpha})+\mathrm{w}_{1} \Delta \bar{\alpha}\right) \frac{\partial \bar{\alpha}}{\partial y_{1}} \mathrm{~d} \bar{s}
$$

and one concludes by the local energy balance (38) that $\bar{J}_{\overline{\mathcal{C}}}=\bar{J}_{\overline{\mathcal{C}}^{\prime}}$. The proof that the equality remains true even if the path are not well ordered is left to the reader. Let us note that this invariance property remains true for more general bulk energy density than (41) because the proof is essentially based on the variational character of the damage evolution law.

The second fundamental property requires an extra assumption which is stated here for future reference.

Hypothesis 4 We assume that, at each time and almost everywhere, $\partial \bar{\alpha} / \partial y_{1} \leq 0$.

Remark 2 The condition $\partial \bar{\alpha} / \partial y_{1} \leq 0$ is automatically satisfied when the crack propagates by virtue of the irreversibility condition and (37). Therefore it is sufficient that the damage remains constant in the neighborhood of the crack tip when the crack does not propagate in order that the condition remains true at every time.

That leads to the

Proposition 4 Under Hypothesis 4, the path integral is a decreasing function of the path, i.e.

$$
\overline{\mathcal{C}} \geq \overline{\mathcal{C}}^{\prime} \quad \Longrightarrow \quad \bar{J}_{\overline{\mathcal{C}}} \leq \bar{J}_{\overline{\mathcal{C}}^{\prime}}
$$

Proof It suffices to start from (42). Then the inner damage criterion (36) and Hypothesis 4 give the desired inequality.

The last property consists in calculating the path integral for large paths.

Proposition 5 Let $\overline{\mathcal{C}}_{\rho}$ be the circle of radius $\rho$ centered at $(0,0)$. Then when $\rho$ goes to infinity, one obtains the following limit for $\bar{J}_{\overline{\mathcal{C}}_{\rho}}$ :

$$
\bar{J}_{\infty}:=\lim _{\rho \rightarrow \infty} \bar{J}_{\overline{\mathcal{C}}_{\rho}}=\bar{G}-\bar{G}_{c}
$$

where $\bar{G}$ and $\bar{G}_{c}$ are the rescaled energy release rate and the rescaled dissipated energy given in (29) and (33). 
Proof The circle $\overline{\mathcal{C}}_{\rho}$ is divided into two complementary parts $\overline{\mathcal{C}}_{\rho}^{o}$ and $\overline{\mathcal{C}}_{\rho}^{b}$, the former corresponding to $\theta \in[\pi-$ $1 / \sqrt{\rho},-\pi+1 / \sqrt{\rho}]$ and the latter to $\theta \in(-\pi,-\pi+1 / \sqrt{\rho}) \cup(\pi-1 / \sqrt{\rho}, \pi)$. Accordingly, for large values of $\rho$, the damage field is nil on $\overline{\mathcal{C}}_{\rho}^{o}$ and the contribution of this part to the path integral reads

$$
\bar{J}_{\overline{\mathcal{C}}_{\rho}^{o}}=\int_{\overline{\mathcal{C}}_{\rho}^{o}}\left(\frac{1}{2} \mathrm{~S}_{0} \overline{\boldsymbol{\sigma}} \cdot \overline{\boldsymbol{\sigma}} n_{1}-\bar{\sigma}_{i j} n_{j} \frac{\partial \bar{u}_{i}}{\partial y_{1}}\right) \mathrm{d} \bar{s} .
$$

This corresponds to the usual Rice integral in a pure linear elastic setting Leblond (2000). Using the asymptotic behavior (34) of $\overline{\boldsymbol{\sigma}}$ at infinity and the fact that the missing angular sector tends to 0 when $\rho$ goes to infinity, one obtains at the limit

$$
\lim _{\rho \rightarrow \infty} \bar{J}_{\overline{\mathcal{C}}_{\rho}^{o}}=\frac{1-\nu_{0}^{2}}{E_{0}}\left(\bar{K}_{I}^{2}+\bar{K}_{I I}^{2}\right)=\bar{G} .
$$

On the complementary part $\overline{\mathcal{C}}_{\rho}^{b}$, for large values of $\rho$, one has:

$$
\mathbf{n} \approx-\mathbf{e}_{1}, \quad \overline{\boldsymbol{\sigma}} \approx \mathbf{0}, \quad \bar{\alpha} \approx \alpha_{*} .
$$

Using (31)-(33), one gets at the limit

$$
\lim _{\rho \rightarrow \infty} \bar{J}_{\overline{\mathcal{C}}_{\rho}^{b}}=-\int_{-\infty}^{\infty}\left(\mathrm{w}\left(\alpha_{*}(\zeta)\right)+\frac{1}{2} \mathrm{w}_{1} \alpha_{*}^{\prime}(\zeta)^{2}\right) \mathrm{d} \zeta=-\bar{G}_{c} .
$$

Hence $\lim _{\rho \rightarrow \infty} \bar{J}_{\overline{\mathcal{C}}_{\rho}}=\bar{G}-\bar{G}_{c}$.

3.5 The link with Griffith's law

We are now in a position to conclude by the

Proposition 6 As long as the internal length of the material is small by comparison with the dimensions of the cracked body and provided that the evolution of damage satisfies Hypotheses 1-4, the evolution of the tip of the crack is governed by Griffith's law, i.e. the following conditions hold

$$
\begin{cases}\text { Irreversibility } & : \dot{\ell} \geq 0, \\ \text { Stability } & : G^{\eta} \leq G_{c}^{\eta}, \\ \text { Energy balance } & :\left(G^{\eta}-G_{c}^{\eta}\right) \dot{\ell}=0 .\end{cases}
$$

Proof Let us prove each item of Griffith's law.

1. The irreversibility condition for $\ell$ is a consequence of that for $\alpha$.

2. From Proposition 2, one knows that $J_{0}^{\eta}=\eta \bar{J}_{0} \leq 0$ where $\bar{J}_{0}=\lim _{\rho \rightarrow 0} \bar{J}_{\overline{\mathcal{C}}_{\rho}}$. By Proposition 4 , one knows that $\bar{J}_{\infty} \leq \bar{J}_{0}$. From Proposition 5, one knows that $\bar{J}_{\infty}=\bar{G}-\bar{G}_{c}$. Hence $G^{\eta}=\eta \bar{G} \leq \eta \bar{G}_{c}=G_{c}^{\eta}$.

3. From Proposition 2, one knows that $J_{0}^{\eta} \dot{\ell}=0$. By Proposition 3, one knows that $\bar{J}_{\infty} \dot{\ell}=\bar{J}_{0} \dot{\ell}$. From Proposition 5 , one knows that $\bar{J}_{\infty}=\bar{G}-\bar{G}_{c}$. Hence $G^{\eta} \dot{\ell}=G_{c}^{\eta} \dot{\ell}$.

The proof is complete. 


\section{Conclusion and perspectives}

Let us first recall that the fundamental result stated in Proposition 6 is an asymptotic property. The crack band behaves like a Griffith crack only when the internal length $\eta$ is small compared to any other length. For instance, the result is not true any more during the nucleation stage of the crack. The construction of the law governing crack nucleation (for instance, at the tip of a notch) with this type of gradient damage model is a very important task to which future works will be devoted. Our result does not hold when the tip of the crack reaches the boundary of the body or is close to an inclusion. In particular, the "effective" propagation of crack bands in composite materials where the distance between the heterogeneities is of the same order as the internal length cannot be obtained with the asymptotic method proposed here.

Let us emphasize that the variational character of the damage evolution law is fundamental to make the link with Griffith's law. All the properties (and the definition itself) of the generalized Rice integral are based on the first order stability conditions and the energy balance. Therefore one can suspect that such a result is no longer true if one adopts constitutive laws which are not connected with these energy principles. Especially in our approach, the construction of the surface energy density $G_{c}$ as an intrinsic material parameter is a consequence of the characteristic damage profile not being history dependent. Several proofs are given here in a simplified context but one can expect that they could be extended to a more general one. In particular, an interesting challenge would be to consider anisotropic materials and to find the dependence of the surface energy density on the orientation of the crack.

The link with Griffith's law is made with the sole help of the first order stability conditions and not with the complete stability condition. It is an advantage by comparison with the Gamma-convergence result based on global minimization. One can consider that our analysis reinforces this convergence result and is also in agreement with the result obtained by Francfort et al. (2009) in a one-dimensional setting. On the other hand, it would be interesting to explore all the consequences of the stability condition. In particular, one notes in the numerical simulations of the thermal shock that the cracks are periodically distributed. An interesting challenge is to give a theoretical proof of this global property of periodicity and even to give a method for calculating the period in terms of the parameters of the problem. We expect that it will be achieved by considering second order stability conditions like in Benallal and Marigo (2007), Pham et al. (2011) and Pham and Marigo (2012b).

\section{A Sketch of the proof of Property (24)}

The proof of property (24) for the general class of strongly brittle materials is not available at the present time. Indeed it needs a complete analysis of the singularities at the tip of the crack. Such an analysis requires additional hypotheses on the state function $W$. Consequently, we merely give the proof in the particular case of the damage model (14) given in Example 1. The analysis of the singularities is made near the tip of the crack and hence we use a polar system of coordinates $(r, \theta)$ with pole the tip of the crack, $\theta=0$ corresponding to the axis tangent to the crack. Accordingly the lips of the crack correspond to $\theta= \pm \pi$. Since the time is fixed, the index $t$ is removed from all fields.

Let us denote $v=1-\alpha$, we only consider singular parts of the displacement and damage fields of the following form:

$$
v(r, \theta)=r^{p} V(\theta)+\cdots, \quad \mathbf{u}(r, \theta)=r^{q}\left(U_{r}(\theta) e_{r}+U_{\theta}(\theta) e_{\theta}\right)+\cdots,
$$

where $p$ and $q$ will be called the order of the singularity. In order that the total energy be finite the orders $p$ and $q$ must be such that $p>0$ and $p+q>0$. The damage criterion (17) 1 reads

$$
v^{-3} \mathrm{~S}_{0} \boldsymbol{\sigma} \cdot \boldsymbol{\sigma}-\mathrm{w}_{1}-\mathrm{w}_{1} \eta^{2} \Delta v \leq 0
$$


Since the first term of (45), $v^{-3} \mathrm{~S}_{0} \boldsymbol{\sigma} \cdot \boldsymbol{\sigma}$, is non negative, it cannot be more singular than the two other terms of (45) whose orders are respectively 0 and at least $p-2$. We can merely consider the case $p \leq 2$ because otherwise the proof is trivial. Therefore, the order of $v^{-3} \mathrm{~S}_{0} \boldsymbol{\sigma} \cdot \boldsymbol{\sigma}$ must be greater or equal to $p-2$. This condition is automatically satisfied when the singular displacement field corresponds to a rigid displacement and in such a case $q \in\{0,1\}$. Otherwise $v^{-3} \mathrm{~S}_{0} \boldsymbol{\sigma} \cdot \boldsymbol{\sigma}$ is of order $p+2 q-2$. Hence, in any case $q$ must be non negative.

From the two inequalities $p>0$ and $q \geq 0$, it is easy to check that $\lim _{r \rightarrow 0} \mathrm{I}_{r}=0$. Moreover the only terms in $\mathrm{J}_{r}$ which can give a non null contribution are the gradient damage terms. Since $\nabla \alpha \cdot \nabla \alpha$ and $q_{r} \alpha, 1$ are both of order $2 p-2, \mathrm{~J}_{0}=0$ if $p>1 / 2$. Therefore, it remains to consider the cases when $p \leq 1 / 2$.

Let us prove that even if $q=0$, the order of $v^{-3} \mathrm{~S}_{0} \boldsymbol{\sigma} \cdot \boldsymbol{\sigma}$ is greater than $p-2$. It is true when $q>0$. If $q=0$, then the order of the strains is at least -1 , the order of $\varepsilon_{r r}$ is greater than -1 and the order of the stresses is at least $2 p-1$. Since the stress field $\boldsymbol{\sigma}$ must satisfy the equilibrium equations, assuming that the body forces are not singular, the singular part of $\operatorname{div} \boldsymbol{\sigma}$ must vanish. This is equivalent to introduce an Airy function of order $2 p+1, \operatorname{say} \phi(r, \theta)=r^{2 p+1} F(\theta)$, and to set

$$
\sigma_{r r}=\frac{1}{r^{2}} \frac{\partial^{2} \phi}{\partial \theta^{2}}+\frac{1}{r} \frac{\partial \phi}{\partial r}+\cdots, \quad \sigma_{r \theta}=-\frac{\partial}{\partial r}\left(\frac{1}{r} \frac{\partial \phi}{\partial \theta}\right)+\cdots, \quad \sigma_{\theta \theta}=\frac{\partial^{2} \phi}{\partial r^{2}}+\cdots
$$

From the stress-strain relation $v^{2} E_{0} \varepsilon_{r r}=\left(1-\nu^{2}\right) \sigma_{r r}-\nu(1+\nu) \sigma_{\theta \theta}$, where $E_{0}$ is the Young modulus of the sound material and $\nu$ the (invariable) Poisson ratio, one deduces that $F(\theta)$ must satisfy

$$
0=(1-\nu) F^{\prime \prime}(\theta)+(1-\nu(2 p+1))(2 p+1) F(\theta) .
$$

Since the lips of the crack are stress free, $F$ must satisfy the boundary conditions $F( \pm \pi)=F^{\prime}( \pm \pi)=0$. Therefore, the unique solution is $F=0$, the order of the stresses is greater than $2 p-1$ and hence the order of $v^{-3} \mathrm{~S}_{0} \boldsymbol{\sigma} \cdot \boldsymbol{\sigma}$ is greater than $p-2$.

Writing (45) at the order $p-2$, one deduces that $V$ must be a non null function which satisfies

$$
V^{\prime \prime}+p^{2} V \geq 0 \quad \text { and } \quad V \geq 0 \text { in }(-\pi, \pi), \quad V( \pm \pi)=0 .
$$

Let us prove that $p \geq 1 / 2$. Multiplying the inequality $V^{\prime \prime}+p^{2} V \geq 0$ by $V$, integrating over $(-\pi, \pi)$, integrating by parts the first term and taking into account the boundary conditions lead to

$$
p^{2} \geq \frac{\int_{-\pi}^{\pi} V^{\prime}(\theta)^{2} \mathrm{~d} \theta}{\int_{-\pi}^{\pi} V(\theta)^{2} \mathrm{~d} \theta} \geq \min _{\substack{\varphi \geq 0 \\ \varphi( \pm \pi)=0}} \frac{\int_{-\pi}^{\pi} \varphi^{\prime}(\theta)^{2} \mathrm{~d} \theta}{\int_{-\pi}^{\pi} \varphi(\theta)^{2} \mathrm{~d} \theta}=\frac{1}{4},
$$

where the last equality is a classical result the proof of which is left to the reader. Moreover, all inequalities become equalities in (47) if and only if $p=1 / 2$ and $V(\theta)=K \cos (\theta / 2)$ with $K>0$. This matches the classical singularity of the laplacian with Dirichlet boundary conditions on the lips of the crack. In such a case, a straightforward calculation gives

$$
\mathrm{J}_{0}=-\frac{\pi}{4} \mathrm{w}_{1} \eta^{2} K^{2}<0
$$

which completes the proof.

Remark 3 Note that the stresses remain bounded since $p \geq 1 / 2$. Moreover, by virtue of Proposition 2 , J $\dot{\mathrm{\ell}}=0$. That means that the gradient of damage can be singular only when the crack does not propagate. Its singular part is then given by

$$
\nabla \alpha=\frac{K}{2 \sqrt{r}}\left(-\cos \frac{\theta}{2} \mathbf{e}_{r}+\sin \frac{\theta}{2} \mathbf{e}_{\theta}\right)+\text { regular terms, } \quad K \geq 0 .
$$

But $K$ must vanish and hence the singularity disappears when the crack propagates.

\section{References}

Ambrosio, L. and V. Tortorelli: 1990, 'Approximation of functionals depending on jumps by elliptic functionals via $\Gamma$ convergence'. 43(8), 999-1036. 
Benallal, A. and J.-J. Marigo: 2007, 'Bifurcation and stability issues in gradient theories with softening'. Modelling and Simulation in Materials Science and Engineering 15, 283-295.

Bourdin, B.: 2007a, 'Numerical implementation of the variational formulation for quasi-static brittle fracture'. Interfaces and Free Boundaries 9, 411-430.

Bourdin, B.: 2007b, 'The variational formulation of brittle fracture: numerical implementation and extensions'. In: Combescure, A and DeBorst, R and Belytschko, T (ed.): IUTAM Symposium on Discretization Methods for Evolving Discontinuities, Vol. 5 of IUTAM Bookseries. pp. 381-393, Springer.

Bourdin, B., G.-A. Francfort, and J.-J. Marigo: 2008, 'The variationnal approach to fracture'. J. Elasticity 91, 5-148.

Braides, A.: 2002, $\Gamma$-convergence for beginners, Oxford Lecture Series in Mathematics and its Applications 22. Oxford: Oxford University Press.

Comi, C.: 1999, 'Computational modelling of gradient-enhanced damage in quasi-brittle materials'. Mechanics of CohesiveFrictional Materials 4(1), 17-36.

Francfort, G. A., N. Q. Le, and S. Serfaty: 2009, 'Critical points of Ambrosio-Tortorelli converge to critical points of Mumford-Shah in the one-dimensional Dirichlet case'. ESAIM-Control Optimisation and Calculus of Variations 15(3), 576-598.

Germain, P.: 1973, Cours de Mécanique des milieux continus. Masson.

Lagerstrom, P.: 1988, Matched Asymptotic Expansions: Ideas and Techniques, Vol. 76 of Applied Mathematical Sciences. Springer-Verlag.

Leblond, J.-B.: 2000, Mécanique de la rupture fragile et ductile, Collection Études en mécanique des matériaux et des structures. Paris: Editions Lavoisier.

Lorentz, E. and S. Andrieux: 1999, 'A variational formulation for nonlocal damage models'. International Journal of Plasticity 15, 119-138.

Pham, K. and J.-J. Marigo: 2010a, 'Approche variationnelle de l'endommagement : I. Les concepts fondamentaux'. Comptes Rendus Mécanique 338(4), 191-198.

Pham, K. and J.-J. Marigo: 2010b, 'Approche variationnelle de l'endommagement : II. Les modèles à gradient'. Comptes Rendus Mécanique 338(4), 199-206.

Pham, K. and J.-J. Marigo: 2012a, 'From the onset of damage up to the rupture: construction of the responses with damage localization for a general class of gradient damage models'. Continuum Mech. Thermodyn. Doi : 10.1007/s00161011-0228-3.

Pham, K. and J.-J. Marigo: 2012b, 'Stability of homogeneous states with gradient damage models: size effects and shape effects in the three-dimensional setting'. J. Elasticity Doi: 10.1007/s10659-012-9382-5.

Pham, K., J.-J. Marigo, and C. Maurini: 2011, 'The issues of the uniqueness and the stability of the homogeneous response in uniaxial tests with gradient damage models'. J. Mech. Phys. Solids 59(6), 1163-1190. 